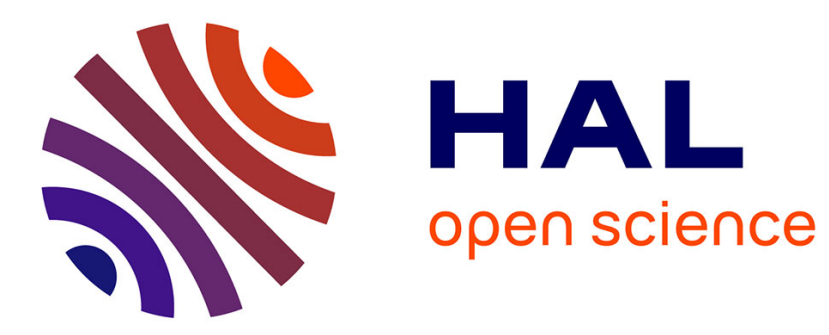

\title{
Numerical simulation of a model of vibrations with joint clearance
}

\author{
Yves Dumont, Laetitia Paoli
}

\section{To cite this version:}

Yves Dumont, Laetitia Paoli. Numerical simulation of a model of vibrations with joint clearance. International Journal of Computer Applications in Technology, 2008, 33 (1), pp.41-53. 10.1504/IJCAT.2008.021884 . hal-01569160

\section{HAL Id: hal-01569160 \\ https://hal.science/hal-01569160}

Submitted on 26 Jul 2017

HAL is a multi-disciplinary open access archive for the deposit and dissemination of scientific research documents, whether they are published or not. The documents may come from teaching and research institutions in France or abroad, or from public or private research centers.
L'archive ouverte pluridisciplinaire HAL, est destinée au dépôt et à la diffusion de documents scientifiques de niveau recherche, publiés ou non, émanant des établissements d'enseignement et de recherche français ou étrangers, des laboratoires publics ou privés. 


\title{
Numerical simulation of a model of vibrations with joint clearance
}

\section{Yves Dumont}

IREMIA, Département de Mathématiques,

Université de la Réunion,

15 avenue René Cassin,

97715 Saint-Denis Messag. 9, France

Fax: +262 262938260

E-mail: Yves.Dumont@univ-reunion.fr

\section{Laetitia Paoli}

LaMUSE, Département de Mathématiques,

Université de Saint-Etienne,

23 rue Paul Michelon,

42023 Saint-Etienne Cedex 2, France

Fax: +33 477485153

E-mail: Laetitia.Paoli@univ-st-etienne.fr

\begin{abstract}
Motivated by the problem of vibrations due to joint clearance between components in complex mechanical systems, we study in this paper the motion of a beam between rigid obstacles. We assume that the material is elastic and the motion is planar. The contact is described with a non-penetration condition, which leads to a model of dynamics with unilateral constraints. We propose a family of fully discretised approximations and their convergence is established. Moreover we present some examples of implementation using either finite element or spline space discretisation.
\end{abstract}

Keywords: convergence; elastic beam; Signorini's conditions; time and space discretisations; vibrations with unilateral constraints.

\section{Introduction}

In several industrial fields, like automotive or aerospace, the assemblies of components in complex mechanical systems are not perfectly achieved and some looseness occurs. Under external forcing, these 'defects' introduce unwanted vibrations and create noise and untimely wear. It is then important to evaluate the consequences of such defects on the global behaviour of the structure in order to propose some 'defect tolerances' which preserve the customer satisfaction (in automotive industry for instance) or the durability of the structures (for assemblies of pipes for instance).

Motivated by such problems, we study in this paper the motion of a beam which is submitted to an external excitation force, which is clamped at its left end and 
which motion is limited by rigid obstacles. The contact is modelled with a non-penetration condition along the obstacles and a Signorini's complementary condition for the displacement and shear stress at the right end of the beam.

More precisely, let us denote by $L$ the length of the beam and by $u(x, t),(x, t) \in \Omega_{T}=(0, L) \times(0, T)$, the vertical displacement. We assume that the material is elastic and the motion is planar. The equilibrium equation is given by

$$
u_{t t}+k^{2} u_{x x x x}=f \text { in } \Omega_{T},
$$

where $f$ is the density of external forces, $k^{2}=\frac{E I}{\rho S}, S$ and $I$ are respectively the surface and the inertial momentum of the section of the beam and $\rho$ and $E$ are respectively the density and the Young's modulus of the material.

The beam is clamped at its left end, i.e.,

$$
u(0, t)=0, \quad u_{x}(0, t)=0 \quad \text { in }(0, T) .
$$

We assume that the vertical displacements are limited by some rigid obstacles, so we define for all $x \in[0, L]$ an interval of admissible positions $\left[g_{1}(x), g_{2}(x)\right]$ such that

$$
u(x, t) \in\left[g_{1}(x), g_{2}(x)\right] \text { in } \bar{\Omega}_{T} .
$$

In order to model both the cases of punctual obstacles or longitudinal ones, we allow $g_{1}(x)$ and $g_{2}(x)$ to take infinite values, i.e., $g_{1}(x) \in \mathbb{R} \cup\{-\infty\}$ and $g_{2}(x) \in \mathbb{R} \cup\{+\infty\}$.

At the right end of the beam, we assume that no moments act, i.e.

$$
u_{x x}(L, t)=0 \text { in }(0, T),
$$

and the Signorini's complementary condition leads to

$$
g_{1}(L) \leq u(L, t) \leq g_{2}(L) \text { in }(0, T),
$$

and

$$
\begin{cases}\sigma(L, t) \geq 0 & \text { if } u(L, t)=g_{1}(L), \\ \sigma(L, t) \leq 0 & \text { if } u(L, t)=g_{2}(L), \\ \sigma(L, t)=0 & \text { if } u(L, t) \in\left(g_{1}(L), g_{2}(L)\right) .\end{cases}
$$

Then equations (1)-(6) can be rewritten as

$$
\left\{\begin{array}{l}
u_{t t}+k^{2} u_{x x x x}=f \quad \text { in } \Omega_{T}, \\
u(x, t) \in\left[g_{1}(x), g_{2}(x)\right] \text { in } \Omega_{T}, \\
u(0, \cdot)=u_{x}(0, \cdot)=u_{x x}(L, \cdot)=0 \text { in }(0, T), \\
u_{x x x}(L, \cdot) \in \partial \psi_{\left[g_{1}(L), g_{2}(L)\right]}(u(L, \cdot)) \text { in }(0, T),
\end{array}\right.
$$

where $\psi_{\left[g_{1}(L), g_{2}(L)\right]}$ denotes the indicator function of the interval $\left[g_{1}(L), g_{2}(L)\right]$ and $\partial \psi_{\left[g_{1}(L), g_{2}(L)\right]}$ is its subdifferential (see Rockafellar, 1970).

Of course, the first equation in (7) should be understood as the equilibrium equation of the system when there is no contact between the beam and the obstacles. Otherwise the constraint equation (3) leads to a reaction force $\mu$, which is a measure on $[0, L] \times[0, T]$ and takes its values in $-\partial \psi_{\left[g_{1}, g_{2}\right]}(u)$, i.e., satisfies the following complementarity condition:

$$
\begin{array}{r}
\frac{\mathrm{d} \mu}{\mathrm{d}|\mu|}(x, t) \in-\partial \psi_{\left[g_{1}(x), g_{2}(x)\right]}(u(x, t)) \\
\mathrm{d}|\mu| \text { a-e on }[0, L] \times[0, T] .
\end{array}
$$

For a more detailed description of the reaction force due to the constraint in the particular case of a single longitudinal obstacle below the beam, see also Ahn and Stewart (2005a, 2005b).

Next we complete the model with initial conditions at $t=0$

$$
u(\cdot, 0)=u_{0}, \quad u_{t}(\cdot, 0)=v_{0} .
$$

In order to obtain a variational formulation of this problem, we define the following functional spaces:

$H=L^{2}(0, L), \quad V=\left\{w \in H^{2}(0, L) ; w(0)=w_{x}(0)=0\right\}$,

$\mathcal{H}=\left\{w \in L^{2}(0, T ; V) ; \quad w_{t} \in L^{2}(0, T ; H)\right\}$,

and the convex set

$$
K=\left\{w \in V ; g_{1}(x) \leq w(x) \leq g_{2}(x) \forall x \in[0, L]\right\} .
$$

We assume that $f \in L^{2}(0, T ; H), v_{0} \in H$ and $u_{0} \in K$. By multiplying the first equation of (7) by a testfunction $v=w-u$ with $w \in \mathcal{H} \cap L^{2}(0, T ; K)$, we obtain the following weak formulation of the problem:

$$
(P)\left\{\begin{array}{l}
\text { Find } u \in \mathcal{H} \cap L^{2}(0, T ; K) \text { such that } \\
\quad-\int_{0}^{T} \int_{0}^{L} u_{t}(x, t)\left(w_{t}(x, t)-u_{t}(x, t)\right) \mathrm{d} x \mathrm{~d} t \\
+k^{2} \int_{0}^{T} \int_{0}^{L} u_{x x}(x, t)\left(w_{x x}(x, t)-u_{x x}(x, t)\right) \mathrm{d} x \mathrm{~d} t \\
\geq \int_{0}^{L} v_{0}(x)\left(w(x, 0)-u_{0}(x)\right) \mathrm{d} x \\
\quad+\int_{0}^{T} \int_{0}^{L} f(x, t)(w(x, t)-u(x, t)) \mathrm{d} x \mathrm{~d} t \\
\forall w \in \mathcal{H} \cap L^{2}(0, T ; K) \operatorname{such} \text { that } w(\cdot, T)=u(\cdot, T) .
\end{array}\right.
$$

For this problem an existence result has been proved by Kuttler and Shillor (2001) by using a penalty method based on the normal compliance approximation of Signorini's condition, when the unilateral constraints equation (3) are created by punctual obstacles at $x=L$, i.e.,

$$
\begin{aligned}
& g_{1}(x)=-\infty, \quad g_{2}(x)=+\infty \quad \forall x \in[0, L), \\
& g_{1}(L)=g_{-}, \quad g_{2}(L)=g_{+},
\end{aligned}
$$

with $g_{-}<0<g_{+}$.

Another existence result has been obtained, still by using a penalty method, by Ahn and Stewart (2005a), in the case of a smooth mapping $g_{1}$ from $[0, L]$ to $\mathbb{R}$, and $g_{2}(x)=+\infty$ for all $x \in[0, L]$, when the external force $f$ does not depend on time. Moreover, they proposed in Ahn and Stewart (2005b) a time-discretisation of the problem, formulated with a complementarity condition involving directly the reaction force due to the constraints (which is a measure on $[0, L] \times[0, T])$.

In a more general case where $g_{1}$ and $g_{2}$ are two mappings from $[0, L]$ to $\overline{\mathbb{R}}$, another existence result has been proved by Dumont and Paoli (2005a, 2006) by using 
a time and space discretisation of $(P)$. More precisely, they use the method of lines, which consists of making first a space discretisation leading to a semi-discretised problem $\left(P_{h}\right)$, which can be rewritten as a second order measure differential inclusion of the same type as those describing rigid bodies dynamics with perfect unilateral constraints. Then, they apply a time-stepping scheme inspired from Newmark's algorithms and Paoli and Schatzman (2002b), which leads to the following family of fully discretised problems:

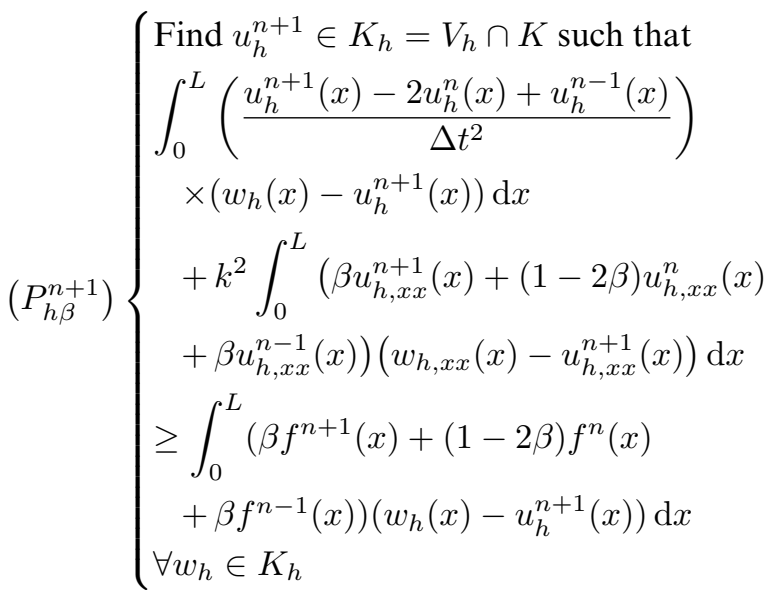

where $\beta$ is a parameter belonging to $[0,1 / 2], f^{n}$ is an approximate value of $f$ at $t_{n}=n \Delta t$ and $V_{h}$ is a finite dimensional subspace of $V$. As usual, there is no need of any impact law in the description of the contact problem as far as the beam is considered as a continuous medium. But, as far as a space discretisation is introduced, some information about the transmission of the velocity at impacts should be added, in order to obtain a closed problem. The choice proposed by Dumont and Paoli (2005a, 2006) corresponds to a vanishing restitution coefficient, which leads to an implicit time discretisation of the semi-dicretised problems $\left(P_{h}\right)$ and finally to the fully discretised problems $\left(P_{h \beta}^{n+1}\right)$. The reader is referred to Dumont and Paoli (2005a) and also Paoli (1993) for a more detailed description of the semi-discretised problems $\left(P_{h}\right)$ and the time-discretisation of $\left(P_{h}\right)$ using Paoli and Schatzman time-stepping scheme for vibro-impact problems.

Let us emphasise that, in the case of contact problems for continuous medium, the chosen value of the restitution coefficient which is needed in the associated space discretised problems $\left(P_{h}\right)$ does not seem to have great influence on the limit when the space step tends to zero, as it has been shown in Paoli and Schatzman (2002a, 2007) from both a numerical point of view and theoretical analysis, in the special case of a slender bar dropped on a rigid foundation.

Furthermore, Dumont and Paoli only have implemented these numerical techniques in the case of punctual obstacles at $x=L$ and they appear to be much more efficient that the penalty approach (for other time-discretisations inspired by Mabrouk (1998) see also Dumont and Paoli (2005b) or Dumont (2002)). In the case of longitudinal obstacles, even if the convergence result remains still valid, the implementation of the discretised problems $\left(P_{h \beta}^{n+1}\right)$ does not seem to be very easy since we look for solutions belonging to $V_{h} \cap K$, i.e., satisfying the constraints on the whole interval $[0, L]$ !

The purpose of this paper is to improve the numerical analysis of Dumont and Paoli (2006) and to extend their previous results from the computational point of view to the case of longitudinal obstacles along the beam, by choosing the set of admissible approximate positions in a more convenient way for implementation. More precisely, we assume that

For all $h>0$, there exists a finite family of points $\left(x_{i}^{h}\right)_{i \in I_{h}}, \operatorname{Card}\left(I_{h}\right)<+\infty$, such that

$\forall x \in[0, L], \quad \forall h>0, \quad \exists i_{h} \in I_{h} / \lim _{h \rightarrow 0} x_{i_{h}}^{h}=x ;$

The mappings $g_{1}, g_{2}:[0, L] \rightarrow \overline{\mathbb{R}}$ satisfy:

$g_{1}(x) \leq-g<0<g \leq g_{2}(x) \quad \forall x \in[0, L]$

with $g \in \mathbb{R}_{+}^{*}$;

For all $x \in[0, L]$ and $j \in\{1,2\}$

$\lim _{h \rightarrow 0} g_{j}\left(x_{i_{h}}^{h}\right)=g_{j}(x)$

where $\left(x_{i_{h}}^{h}\right)_{h>0}$ is the sequence defined at assumption (H1).

Now we replace $K_{h}=V_{h} \cap K$ in $\left(P_{h \beta}^{n+1}\right)$ by

$K_{h}=\left\{w_{h} \in V_{h} ; g_{1}\left(x_{i}^{h}\right) \leq w_{h}\left(x_{i}^{h}\right) \leq g_{2}\left(x_{i}^{h}\right) \forall i \in I_{h}\right\}$.

In other words, the space discretisation is applied also to the constraints since we consider now the unilateral constraints only at the space nodes $\left(x_{i}^{h}\right)_{i \in I_{h}}$.

Since we deal with a fourth order problem with respect to the space derivative, it is not possible to consider a linear space approximation, like the P1 finite element. In fact, we have to consider at least a three order polynomial approximation of the solution. It follows that, in the case of longitudinal obstacles, a function $v_{h} \in V_{h}$ satisfying the unilateral constraints at the nodes, will in general not satisfy them along the subintervals and so will not belong to $K \cap V_{h}$ even if the obstacles are rectilinear.

Furthermore, we can interpret assumption (H3) as a kind of 'discretised continuity property' with respect to the space variable, since the value of $g_{j}$ at $x$ is equal to the limit of the values of $g_{j}$ on the sequence of nodes $\left(x_{i_{h}}^{h}\right)_{h>0}$.

In particular this assumption is immediately satisfied if $g_{1}$ and $g_{2}$ are continuous mappings from $[0, L]$ to $\mathbb{R}$, i.e., in the case of smooth longitudinal obstacles. Nevertheless, assumption (H3) can also be satisfied in case of discontinuous mappings $g_{j}$ taking infinite values: for instance in the case of punctual obstacles, the space network $\left(x_{i}^{h}\right)_{i \in I_{h}, h>0}$ should contain the positions of the punctual obstacles. This last condition does not create any difficulty for the implementation. 
The rest of the paper is organised as follows: in the next section we will study the stablity properties of the proposed discretisation and establish its convergence, then in Section 3 we will present some numerical results obtained with $P 3$ finite element and spline space approximations respectively.

\section{Stability and convergence of the discretisation}

Let us assume from now on that $h>0$ and $V_{h}$ is a finite dimensional subspace of $V$ such that, for all $v \in V$, there exists a sequence $\left(v_{h}\right)_{h>0}$ such that

$$
\left\|v_{h}-v\right\|_{V} \rightarrow_{h \rightarrow 0} 0, \quad v_{h} \in V_{h} \quad \forall h>0 .
$$

We initialise the discretisation of problem $(P)$ by choosing $u_{h}^{0}$ and $u_{h}^{1}$ in $K_{h}$ such that $\left(\left\|u_{h}^{1}\right\|_{V}\right)_{h>0}$ remains bounded and

$$
\lim _{h \rightarrow 0, \Delta t \rightarrow 0}\left\|u_{h}^{0}-u_{0}\right\|_{V}+\left\|\frac{u_{h}^{1}-u_{h}^{0}}{\Delta t}-v_{0}\right\|_{H}=0 .
$$

Let $N \in \mathbb{N}^{*}$ and $\Delta t=T / N$. Then, for all $n \in\{1, \ldots$, $N-1\}$ we solve the following variational inequality:

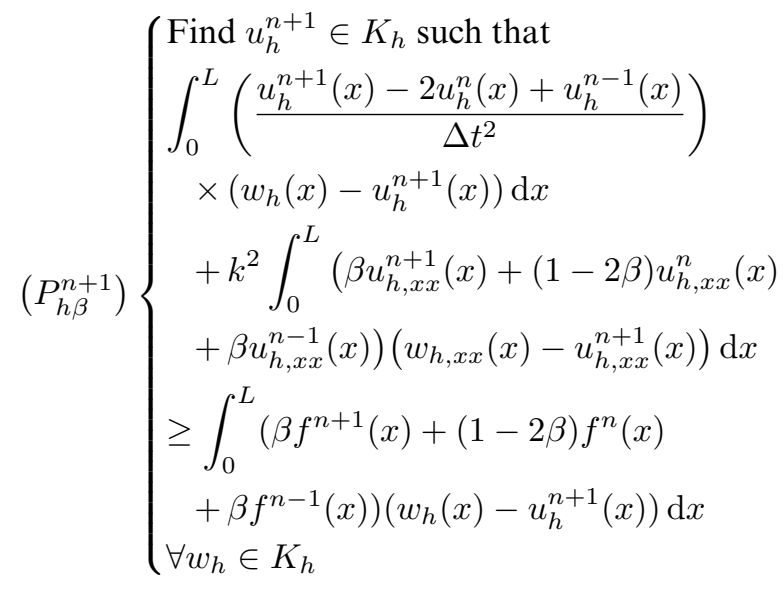

with $K_{h}$ given by equation (8), $\beta \in[0,1 / 2]$ and

$$
f^{n}=\frac{1}{\Delta t} \int_{n \Delta t}^{(n+1) \Delta t} f(\cdot, s) \mathrm{d} s .
$$

Observing that $K_{h}$ is a non-empty closed convex subset of $V_{h}$, we obtain by induction on $n$ that $u_{h}^{n+1}$ is uniquely defined, for all $n \in\{1, \ldots, N-1\}$. Then we introduce the approximate solutions of problem $(P)$ as linear interpolations of the $u_{h}^{n+1}$, i.e.,

$$
u_{h, N}^{\beta}(x, t)=u_{h}^{n} \frac{(n+1) \Delta t-t}{\Delta t}+u_{h}^{n+1} \frac{t-n \Delta t}{\Delta t},
$$

for all $t \in[n \Delta t,(n+1) \Delta t], 0 \leq n \leq N-1$.

First we obtain as in Dumont and Paoli (2006) the following stability property:

Proposition 2.1: Let $\beta \in[0,1 / 2], \quad h>0$ and $\kappa_{h}$ be defined by

$$
\kappa_{h}=\sup _{u_{h} \in V_{h} \backslash\{0\}} \frac{k^{2} \int_{0}^{L}\left|u_{h, x x}(x)\right|^{2} \mathrm{~d} x}{\left\|u_{h}\right\|_{H}^{2}} .
$$

Let $\alpha \in(0,1)$ and $N_{h} \in \mathbb{N}^{*}$ be such that

$$
\begin{aligned}
& N_{h} \geq T+1 \text { if } \beta=1 / 2, \\
& \frac{T}{N_{h}}<\min \left(\sqrt{\frac{2(1-\alpha)}{\kappa_{h}(1-2 \beta)}}, \alpha\right) \text { if } \beta \in[0,1 / 2) .
\end{aligned}
$$

Then there exists a constant depending only on the data, $C\left(f, u_{0}, v_{0}\right)$, such that for all $h>0$ and for all $N \geq N_{h}$ (i.e., $\Delta t \leq \Delta t_{h}=\frac{T}{N_{h}}$ )

$$
\left\|\frac{u_{h}^{n+1}-u_{h}^{n}}{\Delta t}\right\|_{H}^{2}+\left\|u_{h}^{n}\right\|_{V}^{2}+\left\|u_{h}^{n+1}\right\|_{V}^{2} \leq C\left(f, u_{0}, v_{0}\right)
$$

for all $n \in\{1, \ldots, N-1\}$, where $\left(u_{h}^{n+1}\right)_{1 \leq n \leq N-1}$ are the solutions of problems $\left(P_{h \beta}^{n+1}\right)_{1 \leq n \leq N-1}$.

It follows that the sequence $\left(u_{h, N}^{\beta}\right)_{h>0, N \geq N_{h}}$ is bounded in $W=\left\{w \in L^{\infty}(0, T ; V), w_{t} \in L^{\infty}(0, T ; H)\right\}$. Applying Simon's lemma (Simon, 1987), we know that $W$ is compactly embedded in $C^{0}\left([0, T], H^{1}(0, L)\right)$. Hence, possibly extracting a subsequence, still denoted $\left(u_{h, N}^{\beta}\right)_{h>0, N \geq N_{h}}$, there exists $u \in W$ such that

$$
\begin{gathered}
u_{h, N}^{\beta} \rightarrow u \text { weakly* in } W \text { and strongly in } \\
C^{0}\left([0, T], H^{1}(0, L)\right) .
\end{gathered}
$$

It follows that $u(\cdot, 0)=u_{0}$. The key point now is to observe that the approximate solutions $\left(u_{h, N}^{\beta}\right)_{h>0, N \geq N_{h}}$ are uniformly Lispchitz continuous with respect to $x$ i.e.,

Lemma 2.2: Let $\beta \in[0,1 / 2], h>0$ and $N_{h}$ be defined by (10). There exists a constant $k\left(f, u_{0}, v_{0}\right)$, depending only on the data, such that for all $h>0$ and for all $N \geq N_{h}$

$$
\begin{aligned}
& \left|u_{h, N}^{\beta}(x, t)-u_{h, N}^{\beta}\left(x^{\prime}, t\right)\right| \\
& \quad \leq k\left(f, u_{0}, v_{0}\right)\left|x-x^{\prime}\right| \quad \forall t \in[0, T], \quad \forall\left(x, x^{\prime}\right) \in[0, L]^{2} .
\end{aligned}
$$

Proof: Let $h>0$ and $N \geq N_{h}$. With the previous stability property we know that

$$
\left\|u_{h}^{n}\right\|_{V}^{2} \leq C\left(f, u_{0}, v_{0}\right) \quad \forall n \in\{1, \ldots, N\} .
$$

Possibly modifying $C\left(f, u_{0}, v_{0}\right)$, this estimate is also valid for $n=0$ since $\left(u_{h}^{0}\right)_{h>0}$ converges strongly to $u_{0}$ in $V$. Thus $u_{h, x}^{n} \in H^{1}(0, L) \subset C^{0}([0, L])$ and

$$
\begin{aligned}
\left|u_{h, x}^{n}(x)\right| & =\left|u_{h, x}^{n}(x)-u_{h, x}^{n}(0)\right| \leq \int_{0}^{x}\left|u_{h, x x}^{n}(s)\right| \mathrm{d} s \\
& \leq \sqrt{x}\left\|u_{h, x x}^{n}\right\|_{H} \leq \sqrt{L C\left(f, u_{0}, v_{0}\right)}
\end{aligned}
$$

for all $x \in[0, L]$, for all $n \in\{0, \ldots, N\}$. Thus all the functions $u_{h}^{n}$ are $\sqrt{L C\left(f, u_{0}, v_{0}\right)}$-Lipschitz continuous on 
$[0, L]$. Let $k\left(f, u_{0}, v_{0}\right)=\sqrt{L C\left(f, u_{0}, v_{0}\right)}$. For all $\left(x, x^{\prime}\right) \in$ $[0, L]^{2}$ and $t \in[0, T]$, we have

$$
\begin{aligned}
& \left|u_{h, N}^{\beta}(x, t)-u_{h, N}^{\beta}\left(x^{\prime}, t\right)\right| \\
& \leq \quad \frac{(n+1) \Delta t-t}{\Delta t}\left|u_{h}^{n}(x)-u_{h}^{n}\left(x^{\prime}\right)\right| \\
& \quad+\frac{t-n \Delta t}{\Delta t}\left|u_{h}^{n+1}(x)-u_{h}^{n+1}\left(x^{\prime}\right)\right| \\
& \quad \leq k\left(f, u_{0}, v_{0}\right)\left|x-x^{\prime}\right|
\end{aligned}
$$

with $n \in\{0, \ldots, N-1\}$ such that $t \in[n \Delta t,(n+1) \Delta t]$.

\section{It follows that}

Proposition 2.3: The limit u belongs to $\mathcal{H} \cap L^{2}(0, T ; K)$.

Proof: We already know that $u \in W$ and $W \subset \mathcal{H}$, so we just have to prove that

$g_{1}(x) \leq u(x, t) \leq g_{2}(x)$ for all $x \in[0, L]$, for a.e $t \in(0, T)$.

Since $H^{1}(0, L) \subset C^{0}([0, L])$, we have $u \in C^{0}\left(\overline{\Omega_{T}}\right)$ and

$$
u_{h, N}^{\beta}(x, t) \rightarrow u(x, t) \quad \forall(x, t) \in \overline{\Omega_{T}} .
$$

Let $(x, t) \in \overline{\Omega_{T}}$, and $\left(x_{i_{h}}^{h}\right)_{h>0}$ be the sequence defined at assumption (H1). Let $h>0$ and $N \geq N_{h}$, where $N_{h}$ is defined by equation (10). We have

$$
\begin{aligned}
u_{h, N}^{\beta}\left(x_{i_{h}}^{h}, t\right)= & u_{h}^{n}\left(x_{i_{h}}^{h}\right) \frac{(n+1) \Delta t-t}{\Delta t}+u_{h}^{n+1}\left(x_{i_{h}}^{h}\right) \frac{t-n \Delta t}{\Delta t} \\
\leq & g_{2}\left(x_{i_{h}}^{h}\right) \frac{(n+1) \Delta t-t}{\Delta t} \\
& +g_{2}\left(x_{i_{h}}^{h}\right) \frac{t-n \Delta t}{\Delta t}=g_{2}\left(x_{i_{h}}^{h}\right)
\end{aligned}
$$

with $n \in\{0, \ldots, N-1\}$ such that $t \in[n \Delta t,(n+1) \Delta t]$. The previous lemma implies that

$$
\begin{aligned}
u_{h, N}^{\beta}(x, t) & \leq u_{h, N}^{\beta}\left(x_{i_{h}}^{h}, t\right)+k\left(f, u_{0}, v_{0}\right)\left|x-x_{i_{h}}^{h}\right| \\
& \leq g_{2}\left(x_{i_{h}}^{h}\right)+k\left(f, u_{0}, v_{0}\right)\left|x-x_{i_{h}}^{h}\right|
\end{aligned}
$$

Then, assumption (H1) and the 'discrete continuity property' (H3) allow us to pass to the limit when $h$ and $\Delta t$ tend to zero and we get

$$
u(x, t) \leq g_{2}(x)
$$

With similar computations we obtain that $u(x, t) \geq g_{1}(x)$, which concludes the proof.

Remark 2.4: In Dumont and Paoli (2005a, 2005b, 2006) the choice $K_{h}=V_{h} \cap K$ implied immediately that the approximate solutions of $(P)$ belong to $L^{2}(0, T ; K)$. In our case here, the approximate solutions $u_{h, N}^{\beta}$ satisfy the unilateral constraints only at the space nodes $\left(x_{i}^{h}\right)_{i \in I_{h}}$ and thus do not belong necessarily to $L^{2}(0, T ; K)$ anymore.
It remains now to prove that $u$ is solution of problem $(P)$. For that purpose we apply the same techniques as in Dumont and Paoli (2005a, 2006). More precisely, we consider $w \in \mathcal{H} \cap L^{2}(0, T ; K)$ such that $w(\cdot, T)=u(\cdot, T)$, and we prove that

$$
\begin{aligned}
& -\int_{0}^{T} \int_{0}^{L} u_{t}(x, t)\left(w_{t}(x, t)-u_{t}(x, t)\right) \mathrm{d} x \mathrm{~d} t \\
& \quad+k^{2} \int_{0}^{T} \int_{0}^{L} u_{x x}(x, t)\left(w_{x x}(x, t)-u_{x x}(x, t)\right) \mathrm{d} x \mathrm{~d} t \\
& \geq \int_{0}^{L} v_{0}(x)\left(w(x, 0)-u_{0}(x)\right) \mathrm{d} x \\
& \quad+\int_{0}^{T} \int_{0}^{L} f(x, t)(w(x, t)-u(x, t)) \mathrm{d} x \mathrm{~d} t .
\end{aligned}
$$

The main steps consist in

- constructing well-suited test-functions $\left(w_{h}^{n}\right)_{1 \leq n \leq N-1}$ - $\quad$ introducing them in the discretised problems $\left(P_{h \beta}^{n+1}\right)$ and performing a discrete integration

- $\quad$ passing to the limit.

The unilateral constraints prevent us from choosing $w_{h}^{n}$ as the projection on $V_{h}$ of an approximate value of $w$ at $t_{n}=n \Delta t$ (let us observe that $w$ is defined as a element of $V$ only for almost every $t$ ) since the projection does not preserve the constraints. So we construct an auxiliary function $w^{\eta, \mu}$ as follows.

Let $\varepsilon \in(0, T / 2)$ and $\phi$ be a $C^{\infty}$-function such that

$$
\left\{\begin{array}{l}
0 \leq \phi(t) \leq 1 \quad \forall t \in[0, T], \\
\phi(t)=0 \quad \forall t \in[T-3 \varepsilon / 2, T], \quad \phi(t)=1 \quad \forall t \in[0, T-2 \varepsilon] .
\end{array}\right.
$$

We denote $\tilde{w}=(1-\phi) u+\phi w$. Let $\eta \in(0, \varepsilon / 2)$ and $\mu \in(0,1)$. We define $w^{\eta, \mu}$ by

$$
\begin{gathered}
w^{\eta, \mu}(\cdot, t)=u(\cdot, t)+\frac{1}{\eta} \int_{t}^{t+\eta}((1-\mu) \tilde{w}(\cdot, s)-u(\cdot, s)) \mathrm{d} s \\
\forall t \in[0, T-\varepsilon / 2] .
\end{gathered}
$$

We can check easily that $w^{\eta, \mu}-u \in C^{0}([0, T] ; V)$, $w_{t}^{\eta, \mu} \in L^{2}(0, T ; H)$ and $w^{\eta, \mu} \in L^{\infty}(0, T ; V) \cap C^{0}([0, T] ;$ $\left.H^{1}(0, L)\right)$. Moreover recalling that $u \in W$ and $W \subset$ $C^{0,1 / 2}([0, L] \times[0, T])($ see Schatzman and Bercovier, 1989) we have

$$
\begin{aligned}
& \left|u(x, t)-\frac{1}{\eta} \int_{t}^{t+\eta} u(x, s) \mathrm{d} s\right| \\
& \quad \leq \frac{1}{\eta} \int_{t}^{t+\eta}|u(x, t)-u(x, s)| \mathrm{d} s \leq \frac{2 C_{0} \sqrt{\eta}}{3}
\end{aligned}
$$

for all $t \in[0, T-\varepsilon / 2]$ and $x \in[0, L]$, where $C_{0}$ is the Hölder continuity coefficient of $u$. Thus

$$
\begin{aligned}
(1-\mu) g_{1}\left(x_{i}^{h}\right)-\frac{2 C_{0} \sqrt{\eta}}{3} & \leq w^{\eta, \mu}\left(x_{i}^{h}, t\right) \\
& \leq(1-\mu) g_{2}\left(x_{i}^{h}\right)+\frac{2 C_{0} \sqrt{\eta}}{3}
\end{aligned}
$$


for all $t \in[0, T-\varepsilon / 2]$ and for all $i \in I_{h}, h>0$, with the convention that $\alpha g_{j}\left(x_{i}^{h}\right)+\beta=g_{j}\left(x_{i}^{h}\right)$ for all $\alpha>0$ and $\beta \in \mathbb{R}$, if $g_{j}\left(x_{i}^{h}\right) \in\{-\infty,+\infty\}$. It follows that we can choose $\eta$ small enough such that $w^{\eta, \mu}$ satisfies strictly the constraints on the space network $\left(x_{i}^{h}\right)_{i \in I_{h}, h>0}$. Indeed, let $\eta_{\mu} \in(0, \varepsilon / 2)$ be such that

$$
\frac{2 C_{0} \sqrt{\eta_{\mu}}}{3} \leq \frac{\mu}{2} g
$$

where $g$ is the constant defined at assumption (H2). We obtain

$$
g_{1}\left(x_{i}^{h}\right)+\frac{\mu}{2} g \leq w^{\eta, \mu}\left(x_{i}^{h}, t\right) \leq g_{2}\left(x_{i}^{h}\right)-\frac{\mu}{2} g
$$

for all $t \in[0, T-\varepsilon / 2]$ and for all $i \in I_{h}, h>0$.

Then, for $\Delta t<\varepsilon / 2$ and $n \in\{1, \ldots, N-1\}$, we define

$$
w_{h}^{n}= \begin{cases}u_{h}^{n+1}+Q_{h}\left(w^{\eta, \mu}(\cdot, n \Delta t)-u(\cdot,\right. & n \Delta t)) \\ & \text { if } n \Delta t \leq T-\varepsilon, \\ u_{h}^{n+1} & \text { if } n \Delta t>T-\varepsilon,\end{cases}
$$

where $Q_{h}$ is the projection on $V_{h}$ relatively to the scalar product defined on $V$ by the bilinear form $(u, v) \mapsto$ $k^{2} \int_{0}^{L} u_{x x}(x) v_{x x}(x) \mathrm{d} x$. As in Dumont and Paoli (2006) we prove that

Lemma 2.5: Let $\eta \in\left(0, \eta_{\mu}\right)$, with $\eta_{\mu}$ defined by (11). There exist $h_{1}>0$ and $N_{h}^{\prime} \geq N_{h}$ such that, for all $h \in\left(0, h_{1}\right)$ and for all $N \geq N_{h}^{\prime}$, we have

$$
w_{h}^{n} \in K_{h} \quad \forall n \in\{1, \ldots, N-1\} .
$$

Remark 2.6: Let us emphasise that the crucial point here is that $w^{\eta, \mu}$ satisfies strictly the constraints on the space network $\left(x_{i}^{h}\right)_{i \in I_{h}, h>0}$. The construction of $w^{\eta, \mu}$ is inspired from Schatzman and Bercovier (1989), where the case of longitudinal vibrations of a beam was studied.

Next we introduce $w_{h}=w_{h}^{n}$ in $\left(P_{h \beta}^{n+1}\right), 1 \leq n \leq N-1$. With a discrete integration we obtain

$$
\begin{aligned}
\int_{0}^{L} & \left(\frac{u_{h}^{1}(x)-u_{h}^{0}(x)}{\Delta t}\right)\left(w_{h}^{0}(x)-u_{h}^{1}(x)\right) \mathrm{d} x \\
& +\sum_{n=1}^{N-1} \int_{0}^{L}\left(\beta f^{n+1}(x)+(1-2 \beta) f^{n}(x)\right. \\
& \left.+\beta f^{n-1}(x)\right)\left(w_{h}^{n}(x)-u_{h}^{n+1}(x)\right) \mathrm{d} x \Delta t \\
\leq & \sum_{n=1}^{N-1} k^{2} \int_{0}^{L}\left(\beta u_{h, x x}^{n+1}(x)+(1-2 \beta) u_{h, x x}^{n}(x)\right. \\
& \left.+\beta u_{h, x x}^{n-1}(x)\right)\left(w_{h, x x}^{n}(x)-u_{h, x x}^{n+1}(x)\right) \mathrm{d} x \Delta t \\
& -\sum_{n=1}^{N-1} \int_{0}^{L}\left(\frac{u_{h}^{n}(x)-u_{h}^{n-1}(x)}{\Delta t}\right) \\
& \times\left(\frac{\left(w_{h}^{n}(x)-u_{h}^{n+1}(x)\right)-\left(w_{h}^{n-1}(x)-u_{h}^{n}(x)\right)}{\Delta t}\right) \mathrm{d} x \Delta t
\end{aligned}
$$

and we pass to the limit in each term as $h$ and $\Delta t$ tend to zero (see Dumont and Paoli, 2006 for a detailed proof).

\section{Numerical experiments}

In this last section we present two approaches for the space discretisation. In the first one, we consider the classical P3 finite element, which is the space discretisation used in Dumont and Paoli (2005a, 2005b, 2006). In the second approach, we consider cubic B-spline finite element which is used in Ahn and Stewart (2005b) and which leads to smaller systems but is a little bit more difficult to implement. In both cases we consider a partition of the interval $[0, L]$ into $J$ subintervals of length $h$, i.e., we consider the nodes $x_{0}^{h}=0, x_{i}^{h}=i h, \ldots, x_{J}^{h}=L$.

\subsection{P3 finite element}

We use cubics Hermite polynomial as a basis. More precisely, at each node $x_{i}^{h}$, we associate two Hermite piecewise cubics $\varphi_{2 i-1}$ and $\varphi_{2 i}$ defined by

$$
\begin{aligned}
& \varphi_{2 i-1} \in P_{3}, \quad \varphi_{2 i-1}\left(x_{j}^{h}\right)=\delta_{i j} \text { and } \varphi_{2 i-1}^{\prime}\left(x_{j}^{h}\right)=0 \\
& \text { for } 1 \leq j \leq J \\
& \varphi_{2 i} \in P_{3}, \quad \varphi_{2 i}^{\prime}\left(x_{j}^{h}\right)=\delta_{i j} \text { and } \varphi_{2 i}\left(x_{j}^{h}\right)=0 \\
& \quad \text { for } 1 \leq j \leq J .
\end{aligned}
$$

We introduce the following finite dimensional subspace

$$
V_{h}=\operatorname{span}\left\{\varphi_{1}, \varphi_{2}, \ldots, \varphi_{2 J-1}, \varphi_{2 J}\right\} \subset V .
$$

Thus, for all $w_{h} \in V_{h}$ we have

$$
w_{h}=\sum_{i=1}^{2 J} \lambda_{i} \varphi_{i}
$$

where the coordinates $\bar{\lambda}=\left(\lambda_{i}\right)_{1 \leq i \leq 2 J}$ of $w_{h}$ in the basis $\left(\varphi_{i}\right)_{1 \leq i \leq 2 J}$ are characterised by

$$
\lambda_{2 i-1}=w_{h}\left(x_{i}^{h}\right), \quad \lambda_{2 i}=w_{h}^{\prime}\left(x_{i}^{h}\right) \quad \forall i \in\{1, \ldots, J\} .
$$

Let us observe that $w_{h} \in K_{h}$ if and only if

$$
\bar{\lambda} \in \prod_{i=1}^{J}\left(\left[g_{1}\left(x_{i}^{h}\right), g_{2}\left(x_{i}^{h}\right)\right] \times \mathbb{R}\right) .
$$

So problem $\left(P_{h \beta}^{n+1}\right)$ can be rewritten as follows

$$
\left\{\begin{array}{l}
\text { Find } u_{h}^{n+1}=\sum_{i=1}^{2 J} \mu_{i}^{n+1} \varphi_{i} \text { with } \\
\bar{\mu}^{n+1} \in \mathcal{K}_{h}=\prod_{i=1}^{J}\left(\left[g_{1}\left(x_{i}^{h}\right), g_{2}\left(x_{i}^{h}\right)\right] \times \mathbb{R}\right) \\
\text { such that }\left(\mathcal{M}\left(\frac{\bar{\mu}^{n+1}-2 \bar{\mu}^{n}+\bar{\mu}^{n-1}}{\Delta t^{2}}\right), \bar{\lambda}-\bar{\mu}^{n+1}\right) \\
\quad+\left(\mathcal{S}\left(\beta \bar{\mu}^{n+1}+(1-2 \beta) \bar{\mu}^{n}+\beta \bar{\mu}^{n-1}\right), \bar{\lambda}-\bar{\mu}^{n+1}\right) \\
\geq\left(G^{n}, \bar{\lambda}-\bar{\mu}^{n+1}\right), \quad \forall \bar{\lambda} \in \mathcal{K}_{h}
\end{array}\right.
$$


where ( , ) denotes the Euclidean scalar product of $\mathbb{R}^{2 J}$, $\mathcal{M}$ and $\mathcal{S}$ are the global mass and stiffness matrices and

$$
\begin{aligned}
G_{i}^{n}= & \frac{1}{\Delta t} \int_{0}^{L} \int_{n \Delta t}^{(n+1) \Delta t}(\beta f(x, t+\Delta t)+(1-2 \beta) f(x, t) \\
& +\beta f(x-\Delta t)) \varphi_{i}(x) \mathrm{d} x \mathrm{~d} t
\end{aligned}
$$

for all $i \in\{1, \ldots, 2 J\}$.

We refer the reader to Dumont and Paoli (2005b, 2006) for a more detailed description of matrices $\mathcal{M}$ and $\mathcal{S}$. Let us observe that we get with this appraoch two degrees of freedom per nodes, which can give big systems.

\section{$3.2 B$-spline finite element}

We now define $V_{h}$ by using a cubic B-spline finite element which requires only one degree of freedom per node. Indeed, it has been shown that the greater connectivity of the B-splines among the elements enables to obtain good accuracy with few elements (see Hollig, 2001).

Typically, the cubic spline shape functions over the sub-interval $\left[x_{i}^{h}, x_{i+1}^{h}\right](i=0, \ldots, J-1)$ are defined by

$$
\left\{\begin{array}{l}
B_{i-1}(x)=\frac{1}{6}\left(1-\frac{x-x_{i}^{h}}{h}\right)^{3} \\
B_{i}(x)=\frac{1}{6}\left(4-3 \frac{x-x_{i}^{h}}{h}+3\left(1-\frac{x-x_{i}^{h}}{h}\right)^{2}\right. \\
\left.\quad-3\left(1-\frac{x-x_{i}^{h}}{h}\right)^{3}\right) \\
B_{i+1}(x)=\frac{1}{6}\left(1+3 \frac{x-x_{i}^{h}}{h}+3\left(\frac{x-x_{i}^{h}}{h}\right)^{2}\right. \\
\left.\quad-3\left(\frac{x-x_{i}^{h}}{h}\right)^{3}\right), \\
B_{i+2}(x)=\frac{1}{6}\left(\frac{x-x_{i}^{h}}{h}\right)^{3} .
\end{array}\right.
$$

So, taking into account the boundary conditions at $x=0$, we consider the following basis $\left\{\Phi_{1}, \ldots, \Phi_{J+1}\right\}$

$\Phi_{1}=2 B_{-1}-B_{0}+2 B_{1}, \quad \Phi_{2}=B_{2}, \ldots \Phi_{J+1}=B_{J+1}$,

and we define $V_{h}=\operatorname{span}\left\{\Phi_{1}, \ldots, \Phi_{J+1}\right\}$. Then, for all $w_{h} \in V_{h}$ we have

$$
w_{h}=\sum_{i=1}^{J+1} \lambda_{i} \Phi_{i}
$$

where $\bar{\lambda}=\left(\lambda_{i}\right)_{1 \leq i \leq J+1}$ are the coordinates of $w_{h}$ associated to the basis $\left(\Phi_{i}\right)_{1 \leq i \leq J+1}$. We may observe that here $w_{h} \in K_{h}$ if and only if

$$
\mathcal{D} \bar{\lambda} \in \prod_{i=1}^{J}\left[g_{1}\left(x_{i}^{h}\right), g_{2}\left(x_{i}^{h}\right)\right],
$$

where $\mathcal{D}$ is the following $(J+1) \times J$ rectangular matrix

$$
\mathcal{D}=\frac{1}{6}\left(\begin{array}{ccccccc}
7 & 1 & 0 & \cdots & & \cdots & 0 \\
2 & 4 & 1 & 0 & \cdots & & \vdots \\
0 & 1 & 4 & \ddots & \ddots & & \\
& \ddots & \ddots & \ddots & 1 & 0 & \vdots \\
\vdots & & 0 & 1 & 4 & 1 & 0 \\
0 & & \cdots & 0 & 1 & 4 & 1
\end{array}\right) .
$$

Except for the first three sub-intervals, the elemental mass and stiffness matrices are given by

$$
\begin{aligned}
& \mathcal{M}_{e}=\frac{h}{5040}\left(\begin{array}{cccc}
20 & 129 & 60 & 1 \\
129 & 1188 & 933 & 60 \\
60 & 933 & 1188 & 129 \\
1 & 60 & 129 & 20
\end{array}\right) \\
& \mathcal{S}_{e}=\frac{k^{2}}{6 h^{3}}\left(\begin{array}{rrrc}
2 & -3 & 0 & 1 \\
-3 & 6 & -3 & 0 \\
0 & -3 & 6 & -3 \\
1 & 0 & -3 & 2
\end{array}\right)
\end{aligned}
$$

Then problem $\left(P_{h \beta}^{n+1}\right)$ can be rewritten as follows

$$
\left\{\begin{array}{l}
\text { Find } u_{h}^{n+1}=\sum_{i=1}^{J+1} \mu_{i}^{n+1} \Phi_{i} \text { with } \bar{\mu}^{n+1} \in \mathcal{K}_{h} \text { such that } \\
\quad+\left(\mathcal{\mathcal { S }}\left(\frac{\bar{\mu}^{n+1}-2 \bar{\mu}^{n}+\bar{\mu}^{n-1}}{\Delta t^{2}}\right), \bar{\lambda}-\bar{\mu}^{n+1}\right) \\
\geq\left(G^{n}, \bar{\lambda}-\bar{\mu}^{n+1}\right) \\
\forall \bar{\lambda} \in \mathcal{K}_{h}=\left\{\bar{\lambda} \in \mathbb{R}^{J+1} ; \mathcal{D} \bar{\lambda} \in \prod_{i=1}^{J}\left[g_{1}\left(x_{i}^{h}\right), g_{2}\left(x_{i}^{h}\right)\right]\right\}
\end{array}\right.
$$

where $\mathcal{M}$ and $\mathcal{S}$ are the global mass and stiffness matrices and

$$
\begin{aligned}
G_{i}^{n}= & \frac{1}{\Delta t} \int_{0}^{L} \int_{n \Delta t}^{(n+1) \Delta t}(\beta f(x, t+\Delta t)+(1-2 \beta) f(x, t) \\
& +\beta f(x, t-\Delta t)) \Phi_{i}(x) \mathrm{d} x \mathrm{~d} t
\end{aligned}
$$

for all $i \in\{1, \ldots, J+1\}$.

\subsection{Implementation and simulations}

Using one of the previous finite element approaches, problem $\left(P_{h \beta}^{n+1}\right)$ reduces to

$$
\left\{\begin{array}{l}
\text { Find } \bar{\mu}^{n+1} \in \mathcal{K}_{h} \text { such that } \\
\left(\mathcal{A} \bar{\mu}^{n+1}-\mathcal{F}^{n}, \bar{\lambda}-\bar{\mu}^{n+1}\right) \leq 0 \quad \forall \bar{\lambda} \in \mathcal{K}_{h}
\end{array}\right.
$$

with $\mathcal{A}=\left(\mathcal{M}+\Delta t^{2} \beta \mathcal{S}\right)$ and

$$
\begin{aligned}
\mathcal{F}^{n}= & \left(2 \mathcal{M}-\Delta t^{2}(1-2 \beta) \mathcal{S}\right) \bar{\mu}^{n} \\
& -\left(\mathcal{M}+\Delta t^{2} \beta \mathcal{S}\right) \bar{\mu}^{n-1}+\Delta t^{2} G^{n}
\end{aligned}
$$


Since $\mathcal{A}$ is a positive definite symmetric matrix, equation (14) is equivalent to the following minimisation problem

$$
\bar{\mu}^{n+1}=\operatorname{Argmin}_{\bar{\lambda} \in \mathcal{K}_{h}}\left(\frac{1}{2}(\mathcal{A} \bar{\lambda}, \bar{\lambda})-\left(\mathcal{F}^{n}, \bar{\lambda}\right)\right) .
$$

Observing that the condition $\bar{\lambda} \in \mathcal{K}_{h}$ corresponds to linear constraints, the Linear Quadratic Problem (LQP) (15) can be solved by using the Lagrange multipliers method (see Fortin and Glowinski, 1982) or interior-point methods (see Vanderbei, 1999, for a state of art). In the next computations, we will use Scilab a scientific software developed by ENPC-INRIA (Paris, France) and freely available. In particular, we will use the function 'quadpro', implemented in Scilab, which uses the Lagrange multipliers method to solve linear quadratic problem.

We will consider a steel pipe of length $L=10 \mathrm{~m}$, with an external diameter equal to $1 \mathrm{~cm}$ and a thickness equal to $0.5 \mathrm{~mm}$. Thus $k^{2}=\frac{E I}{\rho S}=282.84 \mathrm{~m}^{4} . \mathrm{s}^{-2}$ where $E=2 \times$ $10^{11} \mathrm{~Pa}$ is the Young's modulus, $\rho=8 \times 10^{3} \mathrm{~kg} / \mathrm{m}^{3}$ is the material density. Let assume that $f(x, t)=\sin 10 t$ for all $(x, t) \in \Omega_{T}$ and the initial data are $u_{0}(x)=0$ and $v_{0}(x)=0$ for all $x \in[0, L]$. In the following computations, we choose $\beta=\frac{1}{2}$ (which leads to unconditional stability) and $\Delta t=0.001 \mathrm{~s}$.
We first choose two smooth convex obstacles described by

$$
\begin{aligned}
& g_{1}(x)=-\left(0.005+\left(0.03 \times(x-L / 2)^{2}\right)\right. \text { and } \\
& g_{2}(x)=-g_{1}(x)
\end{aligned}
$$

for all $x \in[0, L]$. In Figure 1, we show the behaviour of the beam obtained with the P3 and the B-spline finite elements, at different times $t_{k} \in[0.4,0.78]$ such that $t_{k}=0.4+(20 \Delta t) k$, with $k=1, \ldots, 19$.

Then, we consider flat obstacles along the second half of the beam, i.e.,

$$
\begin{aligned}
& g_{1}(x)=\left\{\begin{array}{ll}
-\infty & \forall x \in\left(0, \frac{L}{2}\right), \\
-0.01 & \forall x \in\left[\frac{L}{2}, L\right],
\end{array}\right. \text { and } \\
& g_{2}(x)= \begin{cases}+\infty & \forall x \in\left(0, \frac{L}{2}\right), \\
0.01 & \forall x \in\left[\frac{L}{2}, L\right] .\end{cases}
\end{aligned}
$$

In Figures 2 and 3, we show the behaviour of the whole beam at different times $t_{k} \in[0.5,0.78]$ such that $t_{k}=0.5+$

Figure 1 Convex obstacles $(J=40)$ : displacements of the beam computed at several times with the spline and the P3 finite elements for $\beta=\frac{1}{2}$
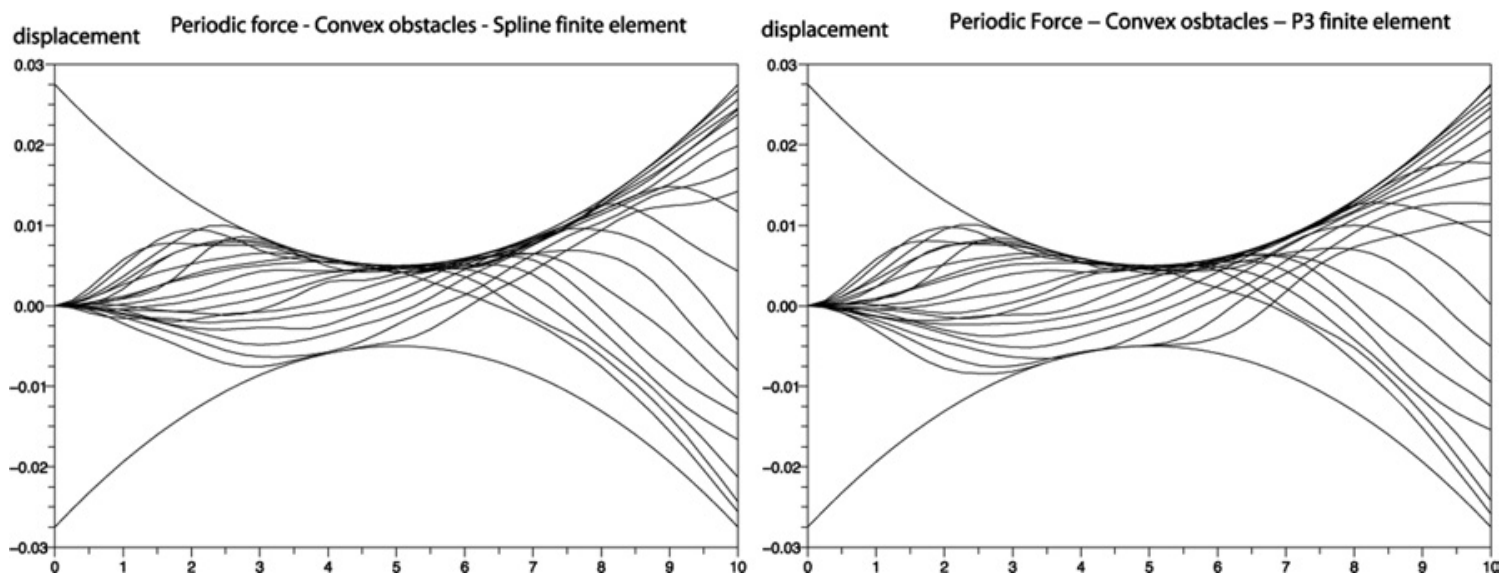

Figure 2 Flat obstacles $(J=40)$ : displacements of the beam computed at several times with the spline and the P3 finite elements for $\beta=\frac{1}{2}$
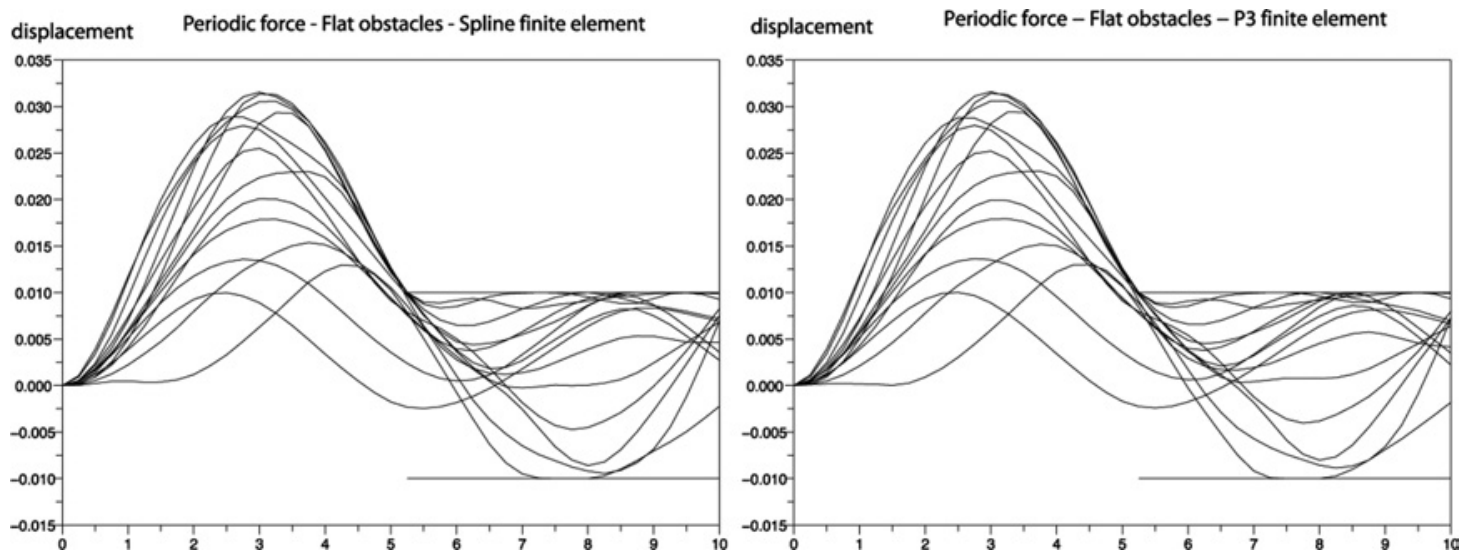
Figure 3 Flat obstacles $(J=80)$ : displacements of the beam computed at several times with the spline and the P3 finite elements for $\beta=\frac{1}{2}$
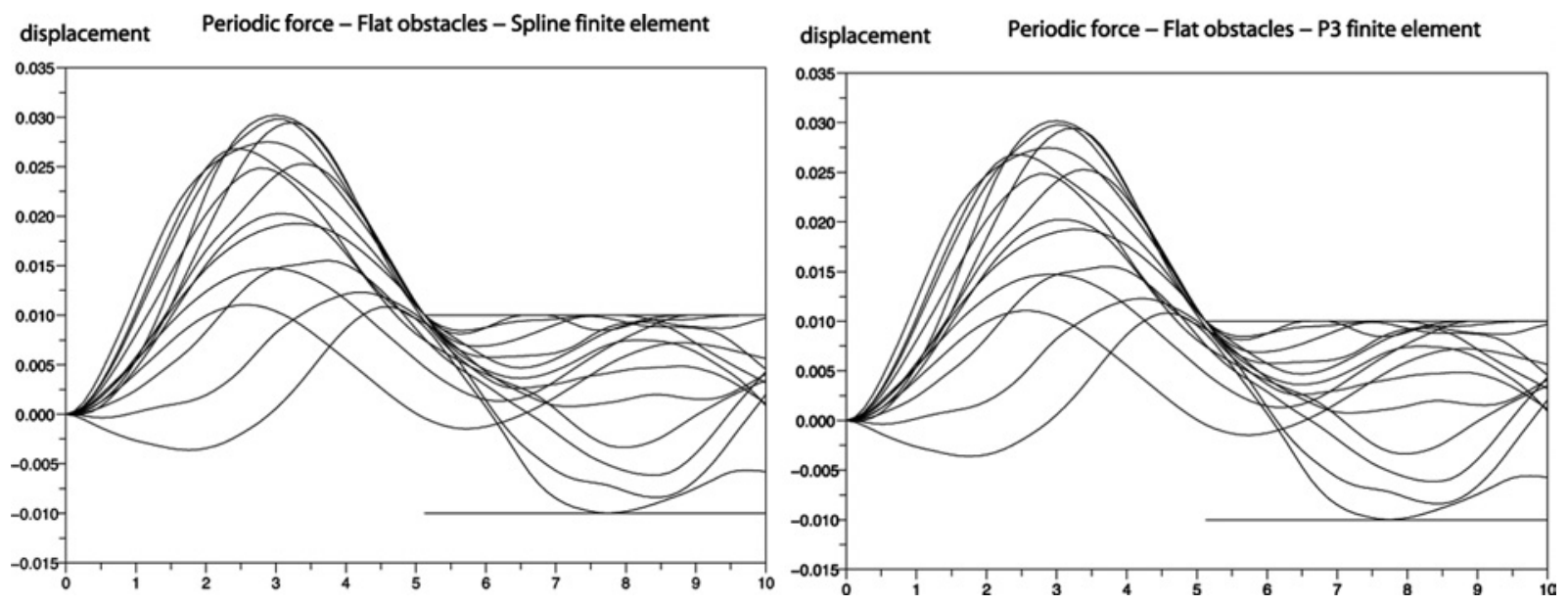

$(20 \Delta t) k$, with $k=1, \ldots, 14$. Note that the behaviour of the beam is slightly different with $J=40$ and $J=80$.

In Table 1, page 15, we present a comparison of the CPU-times (all computations were done on a Mac $2 \mathrm{Ghz}$ Core duo). As expected, the B-spline approach is faster since we have a $J+1$ dimensional quadratic problem instead of a $2 J$ dimensional quadratic problem for the P3 approach. Anyway, for this value of $\Delta t$, i.e., $\Delta t=0.001 \mathrm{~s}$, the computations are relatively fast. Let us emphasise that we can take a rather big time step size since we have unconditional stability for $\beta=\frac{1}{2}$ (see Proposition 2.1). If we choose $\Delta t=10^{-4} \mathrm{~s}$, we obtain a much larger CPU-time. Indeed, in the case of convex obstacles, the P3-approach takes 6351 CPU-time, and the B-spline approach only 1645 CPU-time.

Table 1 Comparison of the CPU-times

\begin{tabular}{lcccc}
\hline & $\begin{array}{c}\text { Convex } \\
\text { obstacle } \\
\text { spline }\end{array}$ & $\begin{array}{c}\text { Convex } \\
\text { obstacle } \\
\text { P3 }\end{array}$ & $\begin{array}{c}\text { Flat } \\
\text { obstacle } \\
\text { spline }\end{array}$ & $\begin{array}{c}\text { Flat } \\
\text { obstacle } \\
\text { P3 }\end{array}$ \\
\hline$J=40$ & & & & \\
CPU time (s) & 40.62 & 124.39 & 37.39 & 103.90 \\
$J=80$ & & & & \\
CPU time (s) & 189.39 & 873.91 & 157.89 & 763.29 \\
\hline
\end{tabular}

With our choice of $K_{h}$, the approximate solutions satisfy the non-penetration conditions at each space nodes but can violate it between two successive nodes. From Lemma 2.2, we already know that the penetration can be estimated from above by $\min _{i \in I_{h}} k\left(f, u_{0}, v_{0}\right)\left|x-x_{i}^{h}\right|=$ $k\left(f, u_{0}, v_{0}\right) \frac{\Delta x}{2}$, where $\Delta x=\frac{L}{J}$ is the space step size. For small values of $\mathrm{J}$, this estimate leads to a quite coarse approximation of the unilateral constraints and it is important to check numerically what happens. As we can see in Tables 2 and 3, the penetration is much more important in the case of a flat obstacles which is not so surprising since $u_{h, N}^{\beta}$ is approximated by a polynomial function of degree 3 , which shape is 'far' from being flat.

Table 2 Convex obstacles - maximum of the penetration computed with the P3 and B-spline approaches

\begin{tabular}{lccc}
\hline & $J=40$ & $J=80$ & $J=160$ \\
\hline$P 3$ & 0.0001076 & 0.0000322 & 0.0000051 \\
$\max _{(x, t) \in \Omega_{T}}$ & & & \\
$\quad\left(g_{1}(x)-u_{h, N}^{\beta}(x, t)\right)^{+}$ & & & \\
$\max _{(x, t) \in \Omega_{T}}$ & 0.0001609 & 0.0000196 & 0.0000046 \\
$\quad\left(u_{h, N}^{\beta}(x, t)-g_{2}(x)\right)^{+}$ & & & \\
$B$-spline & & & \\
$\max _{(x, t) \in \Omega_{T}}$ & 0.0001589 & 0.0000268 & 0.0000044 \\
$\quad\left(g_{1}(x)-u_{h, N}^{\beta}(x, t)\right)^{+}$ & & & \\
$\max _{(x, t) \in \Omega_{T}}$ & 0.0001690 & 0.0000308 & 0.0000050 \\
$\quad\left(u_{h, N}^{\beta}(x, t)-g_{2}(x)\right)^{+}$ & & & \\
\hline
\end{tabular}

Table 3 Flat obstacles - maximum of the penetration computed with the P3 and B-spline approaches

\begin{tabular}{lccc}
\hline$P 3$ & $J=40$ & $J=80$ & $J=160$ \\
\hline $\max _{(x, t) \in \Omega_{T}}\left(g_{1}(x)-u_{h, N}^{\beta}(x, t)\right)^{+}$ & 0.0106 & 0.0037 & 0.00151 \\
$\max _{(x, t) \in \Omega_{T}}\left(u_{h, N}^{\beta}(x, t)-g_{2}(x)\right)^{+}$ & 0.0110 & 0.0038 & 0.00163 \\
$B$-spline & $J=40$ & $J=80$ & $J=160$ \\
$\max _{(x, t) \in \Omega_{T}}\left(g_{1}(x)-u_{h, N}^{\beta}(x, t)\right)^{+}$ & 0.00136 & 0.00034 & 0.000096 \\
$\max _{(x, t) \in \Omega_{T}}\left(u_{h, N}^{\beta}(x, t)-g_{2}(x)\right)^{+}$ & 0.00145 & 0.000279 & 0.000083 \\
\hline
\end{tabular}

In order to complete our numerical study, we now consider the total energy of the system. With $f=0$, as in Ahn and Stewart (2005b), we expect dissipation of energy for the discretised system, although the continuous problem conserves the energy. Thus, we want to investigate numerically the evolution of the energy when $\Delta x$ and $\Delta t$ tend to zero. Indeed, if we choose $\bar{\lambda}=\bar{\mu}^{n-1}$ in equation (12) or equation (13), we obtain $\mathcal{E}_{\beta}^{n+1} \geq \mathcal{E}_{\beta}^{n}$ for all $n \geq 1$, where $\mathcal{E}_{\beta}^{n}$ is the discrete energy given by

$$
\begin{aligned}
\mathcal{E}_{\beta}^{n}= & \left(\bar{\mu}^{n}-\bar{\mu}^{n-1}\right)^{T}\left(\frac{1}{\Delta t^{2}} \mathcal{M}+\beta \mathcal{S}\right)\left(\bar{\mu}^{n}-\bar{\mu}^{n-1}\right) \\
& +\left(\bar{\mu}^{n}\right)^{T} \mathcal{S} \bar{\mu}^{n-1} .
\end{aligned}
$$


As an example, we will consider $u_{0}(x)=0.005 x^{2}, v_{0}=-x$ and

$$
g_{1}(x)=-\left(0.2+\left(0.3 \times(x-L / 2)^{2}\right)\right), \quad g_{2}(x)=-g_{1}(x)
$$

for all $x \in[0, L]$. In Figures 4 and 5, we show the evolution of the energy for different values of the time step and the space step for $\beta=\frac{1}{2}$. Even if the Newmark's scheme with $\beta=\frac{1}{2}$ is known to be dissipative we can observe in Figures 4 and 5 that the energy remains constant between two successive impacts and is decreasing only at impacts.

It seems also interesting to compare the previous results in the case of an energy conservative scheme (when no impacts occur), i.e., for $\beta=\frac{1}{4}$, which corresponds to the well-known trapezoidal scheme. Figures 6 and 7 show that

Figure 4 Dissipation of energy for $\beta=\frac{1}{2}$ (B-spline and P3 finite elements approaches, $J=40$ )
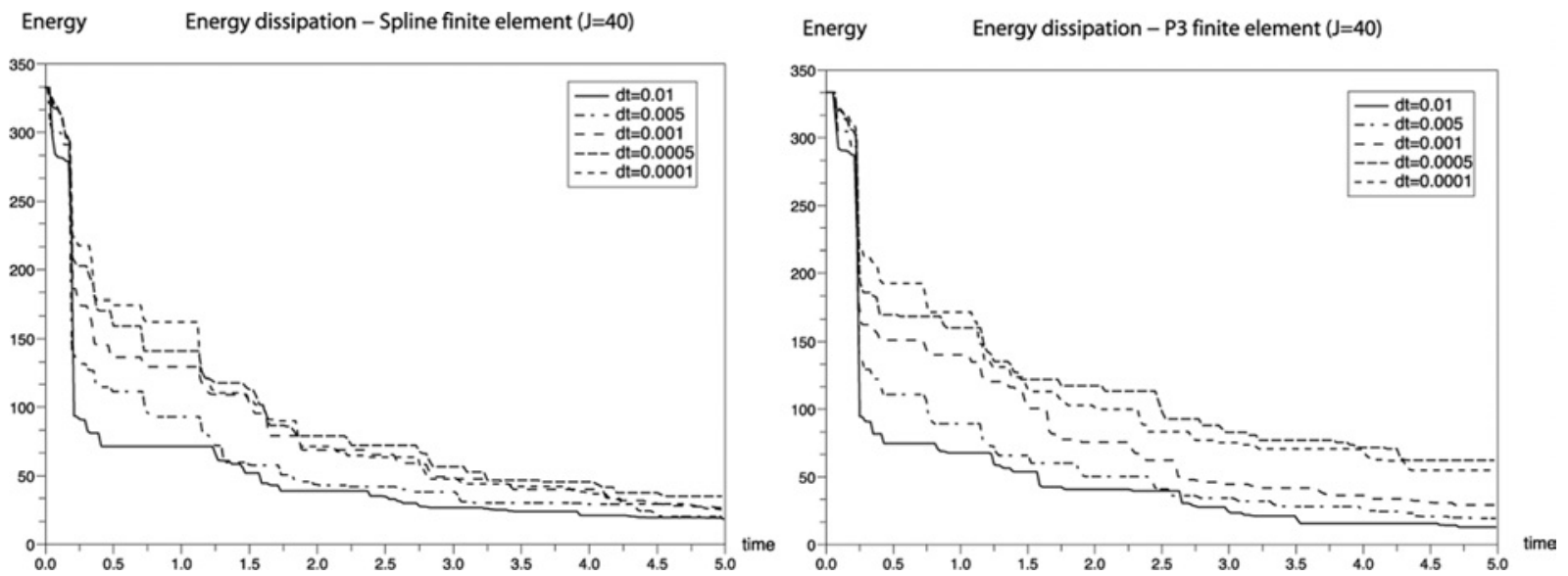

Figure 5 Dissipation of energy for $\beta=\frac{1}{2}$ (B-spline and P3 finite elements approaches, $J=80$ )
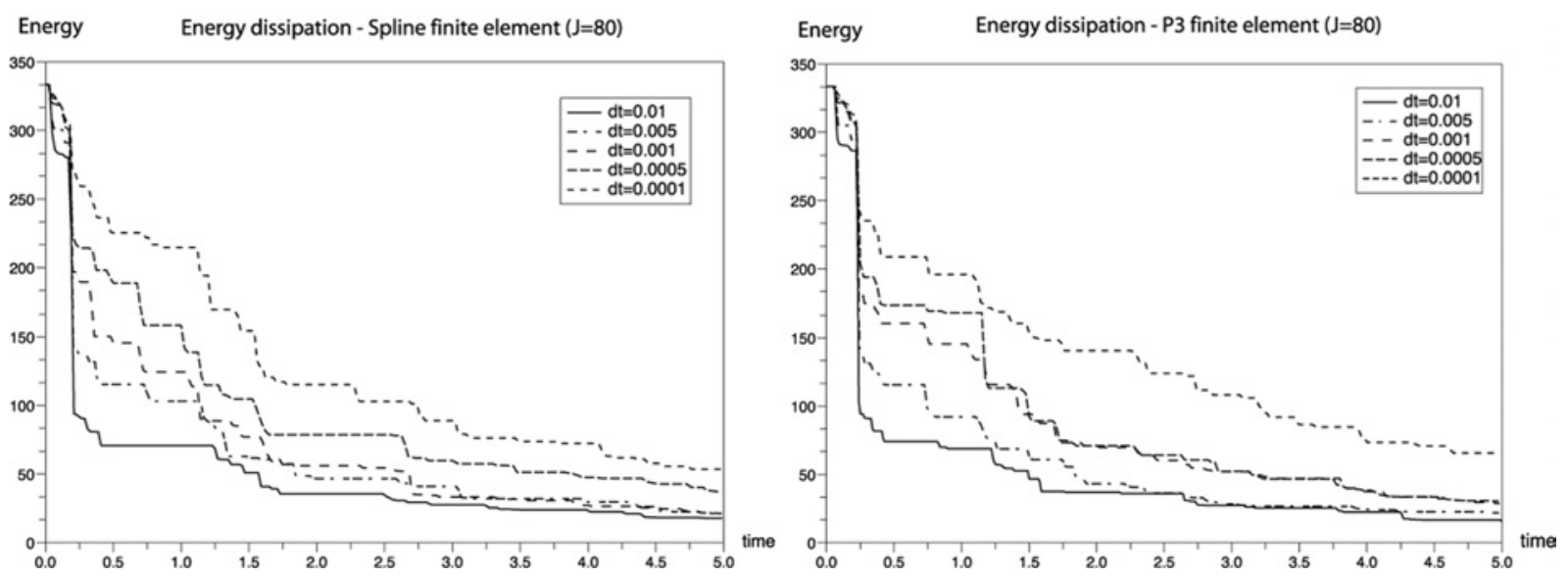

Figure 6 Dissipation of energy with the B-spline finite element for $\beta=\frac{1}{4}$ and $\beta=\frac{1}{2}$ (comparison between $J=40$ and $J=80$ )
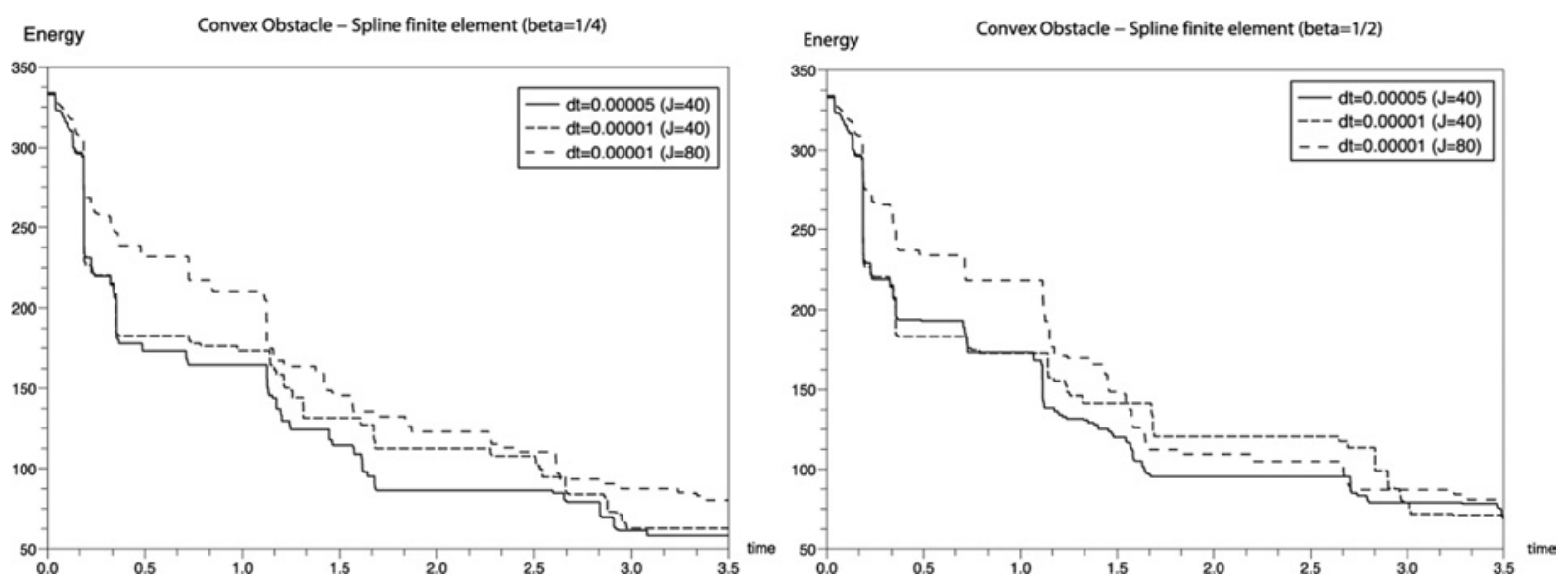
Figure 7 Dissipation of energy with the $\mathrm{P} 3$ finite element for $\beta=\frac{1}{4}$ and $\beta=\frac{1}{2}$ (comparison between $J=40$ and $J=80$ )

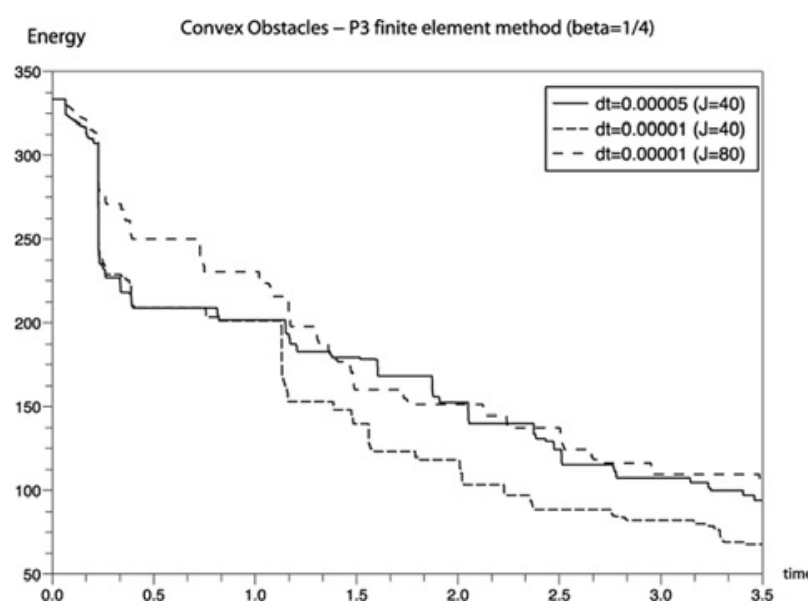

the energy decreases, but only at impacts, as expected. Moreover, we can observe that the behaviour of the energy is quite similar for both values of $\beta, \frac{1}{2}$ and $\frac{1}{4}$. Thus, it seems that the general behaviour of the approximation does not depend on the choice of $\beta$.

Let us recall that for $\beta \neq \frac{1}{2}$, the time-step should satisfy the stability condition $\Delta t \leq \Delta t_{h}$ (see Proposition 2.1). For instance, for $\beta=\frac{1}{4}$ and $J=40$, using the estimate of $\kappa_{h}$ given in the Appendix, we find that $\Delta t_{h} \leq 1.2 \times$ $10^{-4}$ s for the B-spline and $\Delta t_{h} \leq 7 \times 10^{-5}$ s for the P3. Note that for $\Delta t=10^{-5} \mathrm{~s}$ the computations become very expansive (around 48 hours are necessary to complete the computations with the B-spline and the P3 approaches). Thus the importance of the unconditional stability result for $\beta=\frac{1}{2}$.

We also obtain the same qualitative results as Ahn and Stewart (2005b) and we may expect that energy conservation will hold at the limit.

\section{References}

Ahn, J. and Stewart, D.E. (2005a) An Euler-Bernoulli Beam with Dynamic Contact: Penalty Approximation and Existence, Computational Mathematics technical report 163, University of Iowa.

Ahn, J. and Stewart, D.E. (2005b) 'An Euler-Bernoulli beam with dynamic contact: discretisation, convergence and numerical results', SIAM J. Numer. Anal., Vol.43, No. 4, pp.1455-1480.

Dumont, Y. (2002) 'Vibrations of a beam between stops: numerical simulations and comparison of several numerical schemes', Math. Comput. Simul., Vol. 60, Nos. 1-2, pp.45-83.

Dumont, Y. and Paoli, L. (2005a) 'Vibrations of a beam between stops: convergence and implementation of a fully discretised approximation', Proceedings of the Eccomas Thematic Conference "Multibody Dynamics 2005”, CD Rom.

Dumont, Y. and Paoli, L. (2005b) 'Simulations of beam vibrations between stops: comparison of several numerical approaches', Proceedings of the Fifth EUROMECH Nonlinear Dynamics Conference (ENOC-2005), CD Rom.

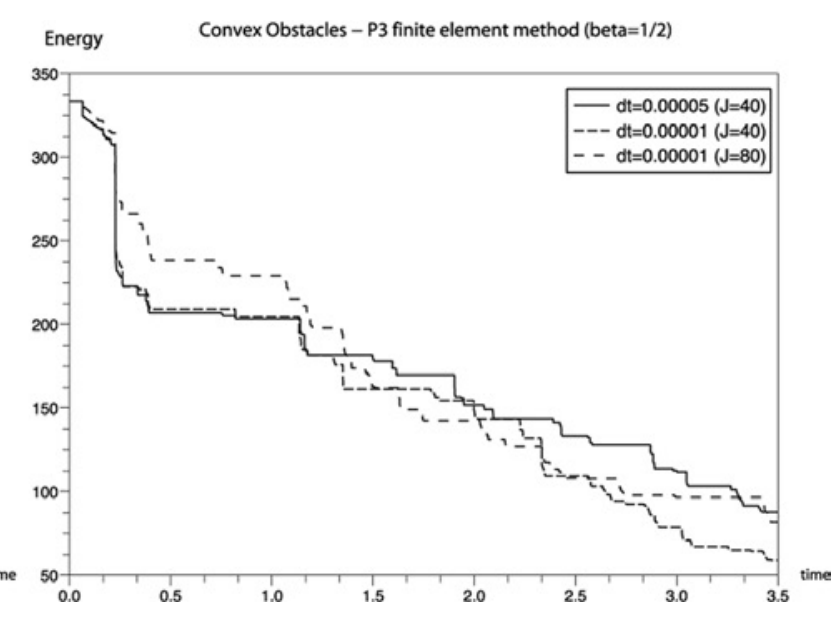

Dumont, Y. and Paoli, L. (2006) 'Vibrations of a beam between stops: convergence of a fully discretised approximation', ESAIM: Math. Modelling Num. Ana. (M2AN), Vol. 40, No. 4, pp.705-734.

Fortin, A. and Glowinski, R. (1982) Méthodes de Lagrangien augmenté, collection Méthodes Mathématiques de l'Informatique sous la dir. de J.L. Lions, Dunod, Paris.

Kuttler, K. and Shillor, M. (2001) 'Vibrations of a beam between two stops', Dynamics of Continuous, Discrete and Impulsive Systems, Series B, Applications and Algorithms, Vol. 8, pp.93-110.

Hollig, K. (2001) Finite Element with B-Splines, in Frontiers in Applied Mathematics, SIAM, Philadelphia.

Mabrouk, M. (1998) 'A unified variational model for the dynamics of perfect unilateral constraints', Eur. J. Mechanics A/Solids, Vol. 17, pp.819-842.

Paoli, L. (1993) Analyse Numérique de Vibrations Avec Contraintes Unilatérales, PhD Thesis, Université Lyon I.

Paoli, L. and Schatzman, M. (2002a) 'Understanding impact through continuous medium vibrations', in Martins, J. and Monteiro Marques, M. (Eds.): Solid Mechanics and its Applications, Kluwer Academic Publishers, Boston, pp.53-64.

Paoli, L. and Schatzman, M. (2002b) 'A numerical scheme for impact problems I and II', SIAM on Numerical Analysis, Vol. 40, No. 2, pp.702-733, 734-768.

Paoli, L. and Schatzman, M. (2007) 'Numerical simulation of the dynamics of an impacting bar', Computer Methods in Applied Mechanics and Engineering, Vol. 196, pp.2839-2851.

Rockafellar, R.T. (1970) Convex Analysis, Princeton University Press, Princeton.

Schatzman, M. and Bercovier, M. (1989) 'Numerical approximation of a wave equation with unilateral constraints', Math. Comp., Vol. 53, No. 187, pp.55-79.

Simon, J. (1987) 'Compact sets in the space $L^{p}(0, T ; B)$ ', Ann. Mat. Pura Applic., Vol. 146, pp.65-96.

Vanderbei, R.J. (1999) 'LOQO: an interior point code for quadratic programming. Interior point methods', Optim. Methods Softw., Vols. 11-12, Nos. 1-4, pp.451-484. 


\section{Appendix: Estimate of $\kappa(h)$}

Let us first compute $\left(w_{h}, w_{h}\right)_{L^{2}(0, L)}$, i.e.,

$$
\left(w_{h}, w_{h}\right)_{L^{2}(0, L)}=\sum_{i, j=1}^{J} \lambda_{i} \lambda_{j}\left(\Phi_{i}, \Phi_{j}\right)_{L^{2}(0, L)}
$$

where $(\cdot, \cdot)_{L^{2}(0, L)}$ is the standard inner product. In particular, we have

$$
\begin{aligned}
\left(\Phi_{1}, \Phi_{1}\right)_{L^{2}(0, L)}= & \left(2 B_{-1}-B_{0}+2 B_{1}, 2 B_{-1}\right. \\
& \left.-B_{0}+2 B_{1}\right)_{L^{2}(0, L)}=\frac{6588}{5040} \Delta x, \\
\left(\Phi_{1}, \Phi_{2}\right)_{L^{2}(0, L)}= & \left(2 B_{-1}-B_{0}+2 B_{1}, B_{2}\right)_{L^{2}(0, L)}=\frac{2264}{5040} \Delta x, \\
\left(\Phi_{1}, \Phi_{3}\right)_{L^{2}(0, L)}= & \left(2 B_{-1}-B_{0}+2 B_{1}, B_{3}\right)_{L^{2}(0, L)}=\frac{239}{5040} \Delta x, \\
\left(\Phi_{1}, \Phi_{4}\right)_{L^{2}(0, L)}= & \left(2 B_{-1}-B_{0}+2 B_{1}, B_{4}\right)_{L^{2}(0, L)}=\frac{2}{5040} \Delta x .
\end{aligned}
$$

Thus

$$
\begin{aligned}
\left(w_{h}, w_{h}\right)_{L^{2}(0, L)} & 6588 \\
= & \frac{6540}{5040} \lambda_{1}^{2} \Delta x+\frac{2 \times 2264}{5040} \lambda_{1} \lambda_{2} \Delta x+\frac{2 \times 239}{5040} \lambda_{1} \lambda_{3} \Delta x \\
& +\frac{4}{5040} \lambda_{1} \lambda_{4} \Delta x+\frac{2416}{5040} \sum_{j=2}^{J-2} \lambda_{j}^{2} \Delta x+\frac{2 \times 1191}{5040} \\
& \times \sum_{j=2}^{J-2} \lambda_{j} \lambda_{j+1} \Delta x+\frac{2 \times 120}{5040} \sum_{j=2}^{J-2} \lambda_{j} \lambda_{j+2} \Delta x+\frac{2}{5040} \\
& \times \sum_{j=2}^{J-2} \lambda_{j} \lambda_{j+3} \Delta x+\frac{2 \times 1062}{5040} \lambda_{J-1} \lambda_{J} \Delta x \\
& +\frac{2396}{5040} \lambda_{J-1}^{2} \Delta x+\frac{2 \times 60}{5040} \lambda_{J-1} \lambda_{J+1} \Delta x \\
& +\frac{1208}{5040} \lambda_{J}^{2} \Delta x+\frac{2 \times 129}{5040} \lambda_{J} \lambda_{J+1} \Delta x+\frac{20}{5040} \lambda_{J+1}^{2} \Delta x .
\end{aligned}
$$

and

$$
\begin{aligned}
& 10 \sum_{j=1}^{J-2}\left(6 \lambda_{j}+7 \lambda_{j+1}+2 \lambda_{j+2}\right)^{2} \Delta x \\
& =360 \lambda_{1}^{2} h+850 \lambda_{2}^{2} h+530 \lambda_{J-1}^{2} h+40 \lambda_{J}^{2} \Delta x+ \\
& 890 \times \sum_{j=3}^{J-2} \lambda_{j}^{2} \Delta x+2 \times 120 \sum_{j=1}^{J-2} \lambda_{j} \lambda_{j+2} \Delta x+2 \times 420 \lambda_{1} \lambda_{2} \Delta x \\
& \quad+2 \times 140 \lambda_{J-1} \lambda_{J}+2 \times 560 \sum_{j=2}^{J-2} \lambda_{j} \lambda_{j+1} \Delta x
\end{aligned}
$$

Thus

$$
\begin{aligned}
\left(w_{h}, w_{h}\right)_{L^{2}(0, L)} & \\
= & \frac{1}{5040}\left(10 \sum_{j=1}^{J-2}\left(6 \lambda_{j}+7 \lambda_{j+1}+2 \lambda_{j+2}\right)^{2}+6228 \lambda_{1}^{2}\right. \\
& +1566 \lambda_{2}^{2}+2 \times 1844 \times \lambda_{1} \lambda_{2}+2 \times 119 \lambda_{1} \lambda_{3}+2 \lambda_{1} \lambda_{4} \\
& +2 \times 922 \lambda_{J-1} \lambda_{J}+2 \times 129 \lambda_{J} \lambda_{J+1}+2 \times 60 \lambda_{J-1} \lambda_{J+1} \\
& +1866 \lambda_{J-1}^{2}+1168 \lambda_{J}^{2}+20 \lambda_{J+1}^{2}+1526 \\
& \left.\times \sum_{j=3}^{J-2} \lambda_{j}^{2}+2 \times 631 \sum_{j=2}^{J-2} \lambda_{j} \lambda_{j+1}+2 \sum_{j=1}^{J-2} \lambda_{j} \lambda_{j+3}\right) \Delta x .
\end{aligned}
$$

But

$$
\begin{aligned}
& 2\left(10 \lambda_{J-1}+\frac{129}{6} \lambda_{J}+3 \lambda_{J+1}\right)^{2} \\
& =2\left(100 \lambda_{J-1}^{2}+\frac{1849}{4} \lambda_{J}^{2}+9 \lambda_{J+1}^{2}+2 \times 60 \lambda_{J-1} \lambda_{J+1}\right. \\
& \left.\quad+2 \times 129 \lambda_{J} \lambda_{J+1}+2 \times 215 \lambda_{J-1} \lambda_{J}\right), \\
& \quad 631 \times \sum_{j=3}^{J-2}\left(\lambda_{j}+\lambda_{j+1}\right)^{2}=631\left(\lambda_{3}^{2}+\lambda_{J-1}^{2}\right) \\
& \quad+2 \times 631 \times \sum_{j=3}^{J-2} \lambda_{j} \lambda_{j+1}+1262 \sum_{j=4}^{J-3} \lambda_{j}^{2},
\end{aligned}
$$

and

We observe that

$$
\begin{aligned}
10 & \sum_{j=1}^{J-2}\left(6 \lambda_{j}+7 \lambda_{j+1}+2 \lambda_{j+2}\right)^{2} \Delta x \\
= & \sum_{j=1}^{J-2}\left(360 \lambda_{j}^{2}+490 \lambda_{j+1}^{2}+40 \lambda_{j+2}^{2}\right) \Delta x+2 \times 420 \\
& \times \sum_{j=1}^{J-2} \lambda_{j} \lambda_{j+1} \Delta x+2 \times 120 \sum_{j=1}^{J-2} \lambda_{j} \lambda_{j+2} \Delta x+2 \times 140 \\
& \times \sum_{j=1}^{J-2} \lambda_{j+1} \lambda_{j+2} \Delta x,
\end{aligned}
$$

$$
\begin{aligned}
119 & \left(\lambda_{1}+\lambda_{2}+\lambda_{3}\right)^{2}+69\left(\frac{25}{3} \lambda_{1}+3 \lambda_{2}\right)^{2} \\
& +128\left(\sqrt{3} \lambda_{2}+\frac{4}{\sqrt{3}} \lambda_{3}\right)^{2}+\frac{11856}{9} \lambda_{1}^{2}+42 \lambda_{2}^{2} \\
& +\frac{637}{3} \lambda_{3}^{2}=6228 \lambda_{1}^{2}+1166 \lambda_{2}^{2}+2 \times 1844 \times \lambda_{1} \lambda_{2} \\
& +2 \times 119 \lambda_{1} \lambda_{3}+2 \times 631 \lambda_{2} \lambda_{3}+895 \lambda_{3}^{2}, \\
& \times \sum_{j=1}^{J-2}\left(\lambda_{j}+\lambda_{j+3}\right)^{2} \Delta x=\left(\lambda_{1}^{2}+\lambda_{J+1}^{2}+\lambda_{2}^{2}+\lambda_{J}^{2}\right. \\
& \left.+\lambda_{3}^{2}+\lambda_{J-1}^{2}\right) \Delta x+2 \sum_{j=2}^{J-2} \lambda_{j} \lambda_{j+3} \Delta x+2 \sum_{j=4}^{J-2} \lambda_{j}^{2} \Delta x .
\end{aligned}
$$


Then, we get

$$
\begin{aligned}
\left(w_{h}, w_{h}\right)_{L^{2}(0, L)} & \\
= & \frac{1}{5040}\left(10 \sum_{j=1}^{J-1}\left(6 \lambda_{j}+7 \lambda_{j+1}+2 \lambda_{j+2}\right)^{2}\right. \\
& +\sum_{j=1}^{J-2}\left(\lambda_{j}+\lambda_{j+3}\right)^{2}+631 \sum_{j=1}^{J-1}\left(\lambda_{j}+\lambda_{j+1}\right)^{2} \\
& +119\left(\lambda_{1}+\lambda_{2}+\lambda_{3}\right)^{2}+69\left(\frac{25}{3} \lambda_{1}+3 \lambda_{2}\right)^{2} \\
& +128\left(\sqrt{3} \lambda_{2}+\frac{4}{\sqrt{3}} \lambda_{3}\right)^{2}+\frac{3952}{3} \lambda_{1}^{2}+442 \lambda_{2}^{2} \\
& +\frac{637}{3} \lambda_{3}^{2}+\left(\lambda_{1}+\lambda_{4}\right)^{2}+894 \lambda_{4}^{2} \\
& +2\left(10 \lambda_{J-1}+\frac{129}{6} \lambda_{J}+3 \lambda_{J+1}\right)^{2} \\
& +707\left(\lambda_{J-1}+\lambda_{J}\right)^{2}+959 \lambda_{J-1}^{2}+\frac{2819}{4} \lambda_{J}^{2} \\
& \left.+\lambda_{J+1}^{2}+895 \sum_{j=5}^{J-2} \lambda_{j}^{2}\right) \Delta x .
\end{aligned}
$$

From the previous equality, we deduce the following lower bound for $\left(w_{h}, w_{h}\right)_{L^{2}(0, L)}$

$$
\begin{gathered}
\frac{1}{5040}\left(\frac{3952}{3} \lambda_{1}^{2}+442 \lambda_{2}^{2}+\frac{637}{3} \lambda_{3}^{2}+894 \lambda_{4}^{2}+959 \lambda_{J-1}^{2}\right. \\
\left.+\frac{2819}{4} \lambda_{J}^{2}+\lambda_{J+1}^{2}+895 \sum_{j=5}^{J-2} \lambda_{j}^{2}\right) \Delta x
\end{gathered}
$$

In the same manner, we compute $\left(w_{h, x x}, w_{h, x x}\right)_{L^{2}(0, L)}$, that is

$$
\begin{aligned}
& \left(w_{h, x x}, w_{h, x x}\right)_{L^{2}(0, L)} \\
& =\frac{k^{2}}{6 h^{4}}\left(108 \lambda_{1}^{2}-16 \lambda_{1} \lambda_{2}-\lambda_{1} \lambda_{3}+16 \sum_{i=2}^{J-3} \lambda_{i}^{2}-2 \times 9\right. \\
& \quad \times \sum_{i=1}^{J-3} \lambda_{i} \lambda_{i+1}+2 \sum_{i=1}^{J-2} \lambda_{i} \lambda_{i+3}+14 \lambda_{J-2}^{2}-12 \lambda_{J} \lambda_{J-1} \\
& \left.\quad+8 \lambda_{J}^{2}-6 \lambda_{J} \lambda_{J+1}+2 \lambda_{J+1}^{2}\right) \Delta x .
\end{aligned}
$$

Using Cauchy-Schwarz formula, we 'easily' deduce that

$$
\begin{aligned}
\left(w_{h, x x}, w_{h, x x}\right)_{L^{2}(0, L)} & \frac{26 k^{2}}{6 h^{4}}\left(\sum_{i=3}^{J-2} \lambda_{i}^{2}+\frac{253}{52} \lambda_{1}^{2}+\frac{34}{26} \lambda_{2}^{2}+\frac{53}{52} \lambda_{3}^{2}\right. \\
& \left.+\frac{21}{26} \lambda_{J-1}^{2}+\frac{17}{26} \lambda_{J}^{2}+\frac{6}{26} \lambda_{J+1}^{2}\right) \Delta x
\end{aligned}
$$

and from equation (16), we infer that

$$
\left(w_{h, x x}, w_{h, x x}\right)_{L^{2}(0, L)} \leq 5040 \frac{k^{2}}{\Delta x^{4}} \times\left(w_{h}, w_{h}\right)_{L^{2}(0, L)} .
$$

Finally, we obtain

$$
\kappa(h) \leq 5040 \frac{k^{2}}{\Delta x^{4}} .
$$

Remark 3.1: Of course, this is not an optimal upper bound. Anyway, we get a smaller upper bound than with the P3 finite element for which (Dumont and Paoli, 2006) proved that $\kappa(h) \leq \frac{2729160}{37} \frac{k^{2}}{\Delta x^{4}}$. 